\title{
Relationship between postprandial glucose level and carotid artery stiffness in patients without diabetes or cardiovascular disease
}

\author{
Kentaro Watanabe*, Tatsuya Suzuki, Motoshi Ouchi, Kazunari Suzuki, Makoto Ohara, Masao Hashimoto, \\ Hidetoshi Yamashita, Masaru Okazaki, Kazuhito Ishii and Kenzo Oba
}

\begin{abstract}
Background: The aim of this study was to evaluate the relationship between postprandial glucose level and atherosclerosis in patients without diabetes and cardiovascular disease by determining carotid ultrasonographic variables and serum levels of 1,5-anhydroglucitol (1,5-AG).

Methods: The subjects were 72 patients without diabetes and cardiovascular disease being treated for hypertension or dyslipidemia. The clinical characteristics of all subjects, including the serum level of 1,5-AG, which appears to be well suited for monitoring postprandial hyperglycemia, were evaluated after an overnight fast. The average intima-media thickness (IMT) and the average pulsatility index (PI) of the right and left common carotid arteries were determined with high-resolution ultrasonography and used as ultrasonographic variables. The subjects were divided into a Lower 1,5-AG group $(n=36)$ and a Higher 1,5-AG group $(n=36)$. We evaluated the relationship between clinical characteristics and ultrasonographic variables of the carotid artery in both groups.

Results: The average PI in the Lower 1,5-AG group was significantly higher than that in the Higher 1,5-AG group, but the average IMT did not differ between the groups. Linear regression analysis, with the ultrasonographic variables as the dependent variables, with 1,5-AG as the independent variable, and adjusted for other clinical characteristics, showed significant correlation between 1,5-AG and the PI but not between 1,5-AG and IMT.
\end{abstract}

Conclusion: Our results suggest that postprandial hyperglycemia increases carotid artery stiffness, but not morphological change, in patients without diabetes or cardiovascular disease.

Keywords: 1,5-anhydroglucitol, Pulsatility index, Postprandial glucose, Nondiabetic patients

\section{Background}

Postprandial hyperglycemia is a major risk factor for morbidity and mortality due to cardiovascular disease in patients with diabetes $[1,2]$. Furthermore, in persons with impaired glucose tolerance (IGT), postprandial hyperglycemia, but not fasting hyperglycemia, increases the risk of cardiovascular disease [2]. Accordingly, decreasing postprandial hyperglycemia reduces the risk of cardiovascular disease in persons with either IGT [3] or diabetes [2]. We have recently found that brachial artery flow-mediated dilation is reduced after glucose loading and is negatively

\footnotetext{
* Correspondence: kentaro@nms.ac.jp

Department of Internal Medicine (Divisions of Cardiology, Hepatology, Geriatrics, and Integrated Medicine), Nippon Medical School, 1-1-5 Sendagi, Bunkyo-ku, Tokyo 113-8603, Japan
}

correlated with plasma glucose levels even in persons with normal glucose tolerance [4]. Hence, to reduce the risk of cardiovascular disease, the relationship between postprandial hyperglycemia and cardiovascular risk factors, such as atherosclerosis, should be evaluated. In fact, many tools are available for evaluating and predicting cardiovascular risk. For example, noninvasive ultrasonographic techniques can be used to evaluate the severity of vascular damage and to indicate the risk of progression of organ and vascular damage [5,6]. Carotid ultrasonography is a noninvasive and inexpensive examination and is widely available in outpatient clinics. The intima-media thickness (IMT) of the carotid artery is an ultrasonographic variable useful for evaluating vascular morphological changes and predicting cardiovascular disease [7]. In addition, the 
pulsatility index (PI) of the carotid artery is a hemodynamic variable that is easily measured with Doppler ultrasonography and is considered to reflect peripheral aortic stiffness distal to the measurement point [8]. Hence, the IMT and PI of the carotid artery are suitable variables for morphological and functional assessment of carotid atherosclerosis.

The aim of the present study was to evaluate the relationship between postprandial glucose levels and atherosclerosis in patients without diabetes or cardiovascular disease by determining the IMT and PI of the common carotid artery (CCA).

\section{Methods}

\section{Study subjects}

The subjects were 72 patients ( 24 men and 48 women; average age, $69.4 \pm 10.7$ years) without diabetes or cardiovascular disease being treated for hypertension or dyslipidemia at the outpatient clinic of our division. All subjects were ambulatory and were free of anorexia or stress conditions possibly affecting glycemic conditions. All subjects were considered not to have diabetes because they met none of the following criteria in the past: 1) fasting plasma glucose level $\geq 7.0 \mathrm{mmol} / \mathrm{L}$; 2) 2-hour value $\geq 11.1 \mathrm{mmol} / \mathrm{L}$ on the 75 -g oral glucose tolerance test; 3) casual plasma glucose level of $\geq 11.1 \mathrm{mmol} / \mathrm{L}$; and 4) A1C (NGSP) $\geq 6.5 \%$. The glycemic conditions of all subjects were stable from the time of a non-diabetes diagnosis to the start of the study period.

[9]. Furthermore, subjects were excluded on the basis of the following criteria: pregnancy, previous gastrectomy, anemia, severe illness, serum creatinine $\geq 114.92$ $\mu \mathrm{mol} / \mathrm{L}$, urine protein test $>1+$ (equivalent to $>0.3 \mathrm{~g} / \mathrm{L}$ ), renal glucosuria, liver cirrhosis, chronic hepatitis, and the use of drugs, such as oral hypoglycemic agents, steroids, and traditional Chinese herbal medicines.

\section{Study design Informed consent and ethics regulations}

The study design was approved by the Ethics Committee of Nippon Medical School and carried out in accordance with the principles of the Declaration of Helsinki. Before the start of the study, written informed consent was obtained from all subjects after they had received a clear explanation of the study protocol.

\section{Measurement of carotid ultrasonographic variables}

The IMT and PI of CCA were determined as carotid ultrasonographic variables as previously reported [10]. Carotid ultrasonographic measurements were performed with a high-resolution ultrasonographic scanner and a linear-array $8-\mathrm{MHz}$ transducer (SSA-350A, Toshiba Medical Systems, Co., Ltd, Tokyo, Japan). The IMT was measured at a total of 4 segments clearly visualized with
B-mode imaging of the near and far walls immediately proximal to the carotid bifurcation in the right and left CCAs. The average of the 4 IMT measurements was calculated and defined as the IMT [11].

The PI of the CCA was determined with pulse Doppler ultrasonography. Pulse Doppler volume measurements were performed with a maximum angle of less than 60 degrees at the same points where the IMT had been measured. The sample-volume measurement point was placed at the center of CCA flow, and peak systolic flow velocity (PSV), end-diastolic flow velocity (EDV), and time-averaged flow velocity (TAV) were determined on the basis of sample volumes. The PI was calculated as follows: $\mathrm{PI}=(\mathrm{PSV}-\mathrm{EDV}) / \mathrm{TAV}$. The average of values from the right and left CCAs was calculated and defined as the PI [10].

Carotid ultrasonographic measurements were performed by 2 observers. The correlation coefficient for interobserver reproducibility and the variability of measurements, respectively, were $\mathrm{r}=0.896(\mathrm{P}<0.001)$ and $8.0 \%$ for the IMT and $r=0.979(\mathrm{P}<0.001)$ and $5.8 \%$ for the PI.

\section{Measurement of pulse wave velocity}

To evaluate the relationship between PI and vascular stiffness, the pulse wave velocity (PWV) of the study subjects was evaluated with an automated device (form PWV/ABI; Omron Colin Co., Ltd., Tokyo, Japan) immediately after the carotid ultrasonographic variables were measured, as previously reported [12]. We measured and evaluated the average right and left brachial-ankle PWVs (baPWVs) of 58 of 72 study subjects.

\section{Evaluation and classification of postprandial glucose levels}

Postprandial glucose levels were determined on the basis of 1,5-anhydroglucitol (1,5-AG), a major human polyol. From $99 \%$ to $100 \%$ of $1,5-\mathrm{AG}$ is reabsorbed in normoglycemia, but the reabsorption rate decreases significantly in hyperglycemia in approximate proportion to the degree of hyperglycemia above the renal threshold for glucosuria $[13,14]$. Therefore, the serum level of 1,5-AG appears to be well suited for monitoring glucose homeostasis in patients with near-normoglycemia $[15,16]$ or postprandial hyperglycemia without fasting hyperglycemia $[17,18]$. Furthermore, serum 1,5-AG level has been suggested to be realted to microvascular and macrovascular complications [14]. Subjects with conditions, other than postprandial hyperglycemia, that effect serum 1,5-AG level had already been excluded on the basis of exclusion criteria. The serum 1,5-AG level was evaluated as previously reported [19]: after an overnight fast the 1,5-AG level was measured with an enzymatic method, (Lana 1,5-AG Auto Liquid, Nippon Kayaku, Tokyo, Japan) with an automatic clinical analyzer (model 7150, Hitachi High-Technologies Corporation, Tokyo, Japan). The overnight fasting period was defined as the 12 hours after the start of the last meal, 
Table 1 Clinical characteristics of study subjects

\begin{tabular}{|c|c|c|}
\hline Clinical characteristics & Lower 1,5 -AG group $(n=36)$ & Higher $1,5-A G$ group $(n=36)$ \\
\hline Male sex & $13(36.1)$ & $11(30.6)$ \\
\hline Age (years) & $70.9 \pm 10.2$ & $67.9 \pm 11.2$ \\
\hline $\mathrm{BMI}\left(\mathrm{kg} / \mathrm{m}^{2}\right)$ & $23.8 \pm 4.0$ & $24.0 \pm 5.1$ \\
\hline \multicolumn{3}{|l|}{ Smoking habit } \\
\hline None & $20(55.9)$ & $25(69.4)$ \\
\hline Recent & $10(27.8)$ & $6(16.7)$ \\
\hline Current & $6(16.7)$ & $5(13.9)$ \\
\hline Hypertension & $28(77.8)$ & $23(63.9)$ \\
\hline Statin use & $16(44.4)$ & $14(38.9)$ \\
\hline \multicolumn{3}{|l|}{ Blood pressure (mm Hg) } \\
\hline Systolic & $134.3 \pm 16.1$ & $131.0 \pm 12.6$ \\
\hline Diastolic & $79.9 \pm 7.2$ & $81.8 \pm 7.4$ \\
\hline Total cholesterol (mmol/L) & $5.42 \pm 0.59$ & $5.64 \pm 1.14$ \\
\hline HDL cholesterol (mmol/L) & $1.46 \pm 0.43$ & $1.60 \pm 0.43$ \\
\hline Triglycerides (mmol/L) & $1.58 \pm 0.68$ & $1.46 \pm 0.85$ \\
\hline Uric acid $(\mu \mathrm{mol} / \mathrm{L})$ & $317.6 \pm 98.2$ & $342.4 \pm 82.4$ \\
\hline Serum creatinine $(\mu \mathrm{mol} / \mathrm{L})$ & $71.0 \pm 15.7$ & $76.4 \pm 15.6$ \\
\hline $\mathrm{FPG}(\mathrm{mmol} / \mathrm{L})$ & $5.16 \pm 0.40$ & $5.15 \pm 0.43$ \\
\hline $\mathrm{A} 1 \mathrm{C}(\%)$ & $5.66 \pm 0.32$ & $5.57 \pm 0.28$ \\
\hline $1,5-A G(\mu \mathrm{g} / \mathrm{mL})^{* * *}$ & $12.71 \pm 3.54$ & $23.16 \pm 3.82$ \\
\hline
\end{tabular}

Mean \pm SD, $\mathrm{n}(\%)$.

BMI: body-mass index; FPG: fasting plasma glucose; A1C: glycosylated hemoglobin; 1,5-AG: 1,5-anhydroglucitol. ***P $<0.001$.

as previosly reported [20]. On the basis of serum 1,5-AG levels, the subjects were divided into 2 groups: subjects with lower serum 1,5-AG levels (Lower 1,5-AG group; $\mathrm{n}$ $=36$ ), and subjects with higher serum 1,5-AG levels (Higher 1,5-AG group; $\mathrm{n}=36$ ).

\section{Clinical characteristics of study subjects}

The clinical characteristics evaluated were age, sex, bodymass index (BMI), smoking habit, hypertension, statin use, systolic and diastolic blood pressures, and biochemical variables, including 1,5-AG and HbA1c (Japan Diabetes Society [JDS]). Biochemical variables were evaluated after an overnight fast. Serum total cholesterol, high-density lipoprotein (HDL) cholesterol, triglycerides, uric acid, and creatinine were measured with an automatic analyzer. The HbA1C (JDS) was measured with high-performance liquid chromatography (JDS Lot 3). Also, the HbA1c (JDS) was transformed into A1C (NGSP) as follows: A1C (NGSP) = HbA1c (JDS) + 0.4 [21] .

\section{Statistical analysis}

The Mann-Whitney U-test was used to compare clinical characteristics and ultrasonographic variables between the Lower and Higher 1,5-AG groups. Multivariate linear regression analysis was used to identify associations between carotid ultrasonographic variables and serum 1,5AG levels. In this multivariate linear regression analysis, we assumed that ultrasonographic variables were dependent variables and that clinical characteristics, including the 1,5AG level, were independent variables. Data are presented

Table 2 Multivariate linear regression analysis assuming PI as the dependent variable and 1,5-AG as the independent variable

\begin{tabular}{ccc}
\hline Pulsatility index & $\boldsymbol{\beta}$ Coefficient $(95 \%$ confidence interval) & t-value \\
\hline Model 1** & $-0.023(-0.035--0.010)$ & -3.639 \\
Model 2** & $-0.018(-0.030--0.006)$ & -3.023 \\
Model ${ }^{*}$ & $-0.016(-0.029--0.004)$ & -2.637 \\
Model 4* & $-0.017(-0.029--0.004)$ & -2.631 \\
Model 5* & $-0.017(-0.029--0.004)$ & -2.619 \\
Model 6* & $-0.018(-0.031--0.004)$ & -2.607 \\
Model 7* & $-0.017(-0.031--0.004)$ & -2.583
\end{tabular}

Model 1, unadjusted; Model 2, adjusted for age and sex; Model 3, adjusted for Model 2, BMI, and smoking habit; Model 4, adjusted for Model 3 and systolic BP; Model 5, adjusted for Model 4, statin use, and total cholesterol; Model 6, adjusted for Model 5 and serum creatinine; Model 7, adjusted for Model 6 and $\mathrm{A} 1 \mathrm{C}$

${ }^{*} \mathrm{P}<0.05$, ${ }^{* *} \mathrm{P}<0.01$. 

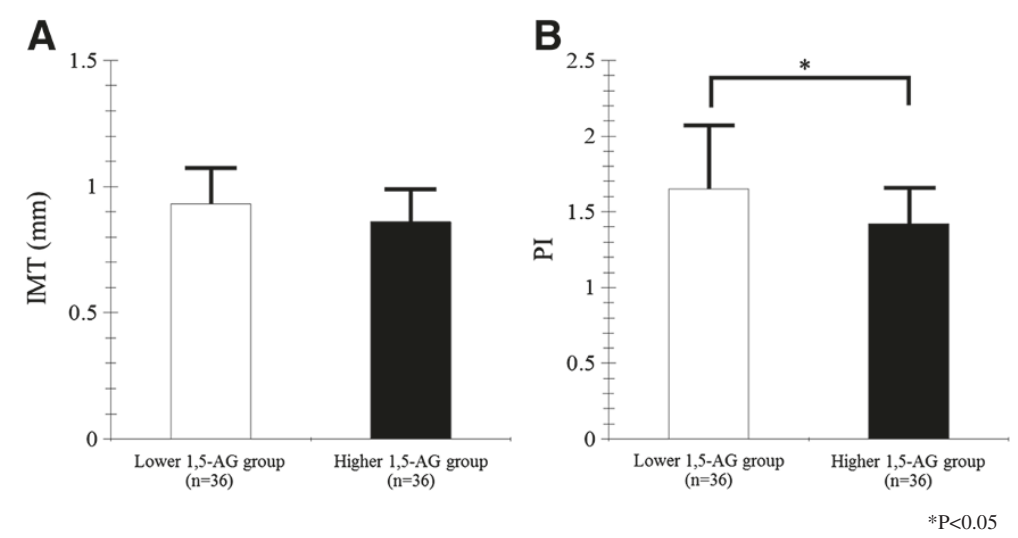

Figure 1 The IMT and PI of subjects in the Lower and Higher 1,5-AG groups. A: Comparison of IMT between the Lower and Higher 1,5-AG groups. B: Comparison of PI between the Lower and Higher 1,5-AG groups.

as means $\pm \mathrm{SD}$ or $\beta$ coefficients (95\% confidence interval, CI). Statistical significance was defined as $\mathrm{P}<0.05$. All analyses were performed with SPSS for Windows Ver. $12.0 \mathrm{~J}$ (IBM SPSS Statistics, IBM Corp., Armonk, NY).

\section{Results}

The average serum 1,5-AG level in the Lower 1,5-AG group $(12.71 \pm 3.54 \mu \mathrm{g} / \mathrm{mL})$ was significantly lower than that in the Higher 1,5-AG group $(23.16 \pm 3.82 \mu \mathrm{g} / \mathrm{mL}$, $\mathrm{P}<0.001$, Table 1). However, no other clinical characteristics differed significantly between the Lower and the Higher 1,5-AG groups.

The average IMT in the Lower 1,5-AG group (0.92 \pm $0.17 \mathrm{~mm}$ ) was higher, but not significantly so, than that in the Higher 1,5-AG group $(0.86 \pm 0.14 \mathrm{~mm} ; \mathrm{P}=0.066$, Figure 1A). On the other hand, the average PI in the Lower 1,5 -AG group $(1.65 \pm 0.43)$ was significantly

Table 3 Multivariate linear regression analysis assuming IMT as the dependent variable and 1,5-AG as the independent variable

\begin{tabular}{ccc}
\hline Intima-media thickness & $\begin{array}{c}\boldsymbol{\beta} \text { Coefficient } \\
\text { (95\% confidence interval) }\end{array}$ & t-value \\
\hline Model 1 & $-0.005(-0.011-0.001)$ & -1.749 \\
Model 2 & $-0.002(-0.007-0.003)$ & -0.683 \\
Model 3 & $-0.002(-0.008-0.003)$ & -0.810 \\
Model 4 & $-0.002(-0.007-0.003)$ & -0.705 \\
Model 5 & $-0.002(-0.007-0.003)$ & -0.785 \\
Model 6 & $-0.001(-0.006-0.005)$ & -0.304 \\
Model 7 & $-0.001(-0.006-0.005)$ & -0.287 \\
\hline
\end{tabular}

Model 1, unadjusted; Model 2, adjusted for age and sex; Model 3, adjusted for Model 2, BMI, and smoking habit; Model 4, adjusted for Model 3 and systolic BP; Model 5, adjusted for Model 4, statin use, and total cholesterol; Model 6, adjusted for Model 5 and serum creatinine;

Model7, adjusted for Model 6 and A1C. higher than that in the Higher 1,5-AG group (1.42 \pm 0.25 , $\mathrm{P}=0.013$, Figure 1B).

Linear regression analysis adjusted for the subjects' clinical characteristics showed significant correlations between 1,5-AG and PI $(\beta=-0.017, \mathrm{t}=-2.583 ; \mathrm{P}=0.012$, Table 2) but not between 1,5-AG and IMT (Table 3 ).

Concerning baPWV in 58 study subjects, the average baPWV in the Lower 1,5-AG group $(1929.0 \pm 489.9 \mathrm{~cm} / \mathrm{s}$, $\mathrm{n}=28$ ) was higher, but not significantly so, than that in Higher 1,5-AG group $(1812.6 \pm 328.2 \mathrm{~cm} / \mathrm{s}, \mathrm{n}=30$; $\mathrm{P}=0.673)$. However, PI was significantly correlated with baPWV $(\mathrm{r}=0.405 ; \mathrm{P}=0.002$, Figure $2 \mathrm{~B})$. In contrast, IMT was significantly, but weakly correlated with baPWV $(\mathrm{r}=0.305 ; \mathrm{P}=0.016$, Figure $2 \mathrm{~A})$.

\section{Discussion}

Our results demonstrate that the serum 1,5-AG level in persons without diabetes or cardiovascular disease is significantly correlated with the PI of the carotid artery but not with the IMT.
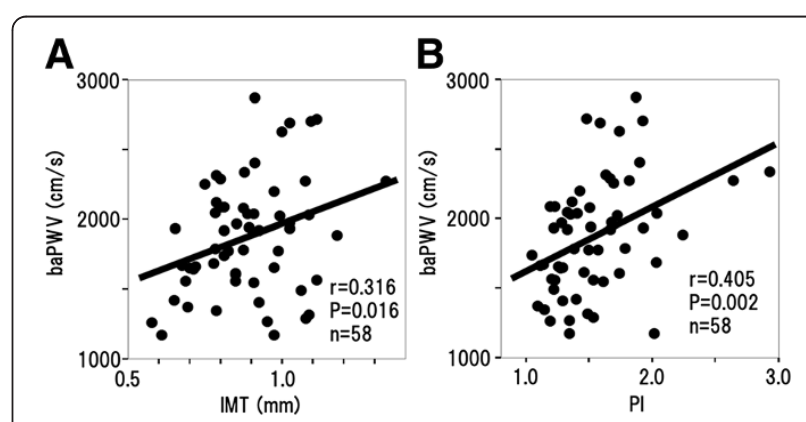

Figure 2 Correlations between baPWV and carotid ultrasonographic variables. A: Correlation between IMT and baPWV. B: Correlation between PI and baPWV. 
Increased vascular stiffness is an early change in atherosclerosis [6]. In particular, several studies have shown that the PI of the carotid artery is significantly correlated with cerebrovascular disease [10,22,23]. Furthermore, the PI of the carotid artery was significantly correlated with Framingham risk scores in subjects with hypertension [24], and systemic artery stiffness is correlated with carotid artery stiffness [25], cardiovascular risk score, and the presence of cardiovascular disease [26]. The present study also found that the PI was significantly correlated with the baPWW. Furthermore, aortic stiffness is an indicator of cardiovascular disease, and high aortic stiffness is correlated with an increased risk of cardiovascular events [27]. On the other hand, reduced stiffness of the CCA is associated with a reduced risk of cardiovascular events [28]. Hence, the relation between carotid artery stiffness and cardiovascular disease suggests that the increased PI of the carotid artery in the present subjects is associated with an increased risk of cardiovascular disease. Our results in subjects without diabetes or cardiovascular disease also suggest that the risk of cardiovascular disease is positively correlated with vascular stiffness, which is induced by postprandial hyperglycemia, rather than with the morphological changes of atherosclerosis. In fact, Li et al. have found that the baPWV is increased in subjects with IGT or newly diagnosed diabetes but not in subjects with normal glucose tolerance or isolated impaired fasting glucose [29]. Furthermore, Huang et al. have found that the vascular stiffness of healthy individuals is significantly and positively correlated with glucose levels 60 minutes after oral glucose challenge [30].

The mechanism of increased vascular stiffness in the absence of the morphological changes of atherosclerosis in the present study remains unclear. However, several previous studies have provided important information regarding the mechanism of how postprandial hyperglycemia increases vascular stiffness. Endothelium-derived nitric oxide (NO) is considered an important factor in the relaxation of vascular smooth muscle cells. The proposed mechanism of relaxation is that NO induces the hyperpolarization of smooth muscle cells by reducing the open probability of Ca-channel-dependent activation of the sarcoplasmatic reticulum which, in turn, decreases $\mathrm{Ca}^{2+}$ influx [31]. Many studies have found that acute hyperglycemia is associated with increased oxidative stress, which inactivates $\mathrm{NO}$ and contributes to endothelial cell injury in vitro [32,33]. In particular, Ceriello et al. have found that glucose levels fluctuating over 24 hours are more deleterious to endothelial function and oxidative stress than are continuously high glucose concentrations in healthy persons or patients with type 2 diabetes [34]. Azuma et al. have shown that repeated fluctuations in glucose or insulin increase monocyte adhesion to the endothelium of the rat thoracic aorta and that stable hyperglycemia or hyperinsulinemia causes less monocyte adhesion [35]. Furthermore, Ge et al. have reported that oxidative stress under intermittently high glucose conditions is significantly greater than that under constantly high glucose conditions in vitro [36]. Such differences have also been found in healthy subjects with normal glucose tolerance [37-39] and in persons with IGT $[38,39]$ or diabetes $[40,41]$. In fact, we have previously demonstrated that oral glucose loading attenuates brachial artery flow-mediated dilation in persons with normal glucose tolerance [4]. Our present findings in subjects without the morphological changes of atherosclerosis suggest that impaired NO bioactivity due to acute glucose elevation in the postprandial state increases carotid vascular stiffness.

The present study had several limitations. First, we could not assess the associations of OGTT plasma glucose levels, with vascular stiffness. This relationship may provide additional information against the results of the present study. Second, we could not assess the relationship between lipid metabolism and carotid artery stiffness. Third, the final meal on the evening before the study was not standardized. 1,5-AG in the body originates mainly from foods and is well absorbed in the intestine. The daily intake of $1,5-\mathrm{AG}$ is approximately $26.8 \mu \mathrm{mol}$ and is independent of food type $(13.4 \mu \mathrm{mol} /$ $100 \mathrm{kcal}$ ) [42]. On the other hand, the serum 1,5-AG level is correlated with the daily urinary glucose excretion level [16]. Therefore, a difference in energy intake or the glycemic index might produce a difference in daily urinary glucose excretion. Finally, the number of subjects in our study was small; therefore, the results of our study should be clarified in a larger population.

\section{Conclusion}

The results of the present study suggest that postprandial hyperglycemia increases carotid vascular stiffness in patients without diabetes or cardiovascular disease. Further prospective study is needed to confirm these results.

\section{Abbreviations \\ IMT: Intima-media thickness; PI: Pulsatility index; IGT: Impaired glucose tolerance; CCA: Common carotid artery; baPWV: Brachial-artery pulse wave velocity; 1,5-AG: 1,5-anhydroglucitol; NO: Nitric oxide.}

\section{Competing interests}

The authors declare that they have no conflict of interest for this study.

\section{Authors' contributions}

KW edited this manuscript. TS and KO contributed to the study conception and design. $\mathrm{MO}$ contributed to the study conception and design. $\mathrm{KS}, \mathrm{MO}$, and $\mathrm{MH}$ participated in data collection, data analysis and interpretation, and drafted the manuscript. HY participated in data collection, data interpretation, and edited the manuscript. MO and KI participated in data correction. All authors read and approved the final manuscript.

\section{Acknowledgements}

The authors are grateful to Masao Okazaki, MD, of the Academic Information Center, The Jikei University School of Medicine, for his careful revision of the English of the manuscript. 
Received: 30 October 2012 Accepted: 22 February 2013

Published: 26 February 2013

\section{References}

1. The DECODE study group on behalf of the European Diabetes Epidemiology Group: Glucose tolerance and mortality: comparison of WHO and American Diabetic Association diagnostic criteria. Lancet 1999, 354:617-621.

2. Ceriello A, Hanefeld M, Leiter L, Monnier L, Moses A, Owens D: Postprandial glucose regulation and diabetic complications. Arch Intern Med 2004 164:2090-2095.

3. Chiasson JL, Josse RG, Gomis R, Hanefeld M, Karasik A, Laakso M: Acarbose treatment and the risk of cardiovascular disease and hypertension in patients with impaired glucose tolerance: the STOP-NIDDM trial. JAMA 2003, 290:486-494.

4. Watanabe K, Oba K, Suzuki T, Ouchi M, Suzuki K, Futami-Suda S, Sekimizu K, Yamamoto N, Nakano H: Oral glucose loading attenuates endothelial function in normal individual. Eur J Clin Invest 2011, 41:465-473.

5. Tomiyama $\mathrm{H}$, Yamashina A: Non-invasive vascular function tests: their pathophysiological background and clinical application. Circ J 2010, 74:24-33.

6. Ter Avest E, Stalenhoef AF, de Graaf J: What is the role of non-invasive measurements of atherosclerosis in individual cardiovascular risk prediction? Clin Sci 2007, 112:507-516.

7. Lorenz MW, von Kegler S, Steinmetz H, Markus HS, Sitzer M: Carotid intimamedia thickening indicates a higher vascular risk across a wide age range: prospective data from the Carotid Atherosclerosis Progression Study (CAPS). Stroke 2006, 37:87-92.

8. Frauchiger B, Bock A, Eichlisberger R, Landmann J, Thiel G, Mihatsch MJ, Jäger $K:$ The value of different resistance parameters in distinguishing biopsy-proved dysfunction of renal allografts. Nephrol Dial Transplant 1995, 10:527-532.

9. Seino Y, Nanjo K, Tajima N, Kadowaki T, Kashiwagi A, Araki E, Ito C, Inagaki N, Iwamoto $Y$, Kasuga M, Hanafusa T, Haneda M, Ueki K: The Committee of the Japan Diabetes Society on the diagnostic criteria of diabetes mellitus. Diabetol Int 2010, 1:2-20.

10. Watanabe K, Suzuki T, Nakano H, Oba K: Usefulness of carotid parameters measured by ultrasonography as a marker of atherothrombotic infarction and lacunar infarction in high-risk elderly people. Geriatr Gerontol Int 2006, 6:87-93.

11. O'Leary DH, Polak JF, Wolfson SK Jr, Bond MG, Bommer W, Sheth S, Psaty BM, Sharrett AR, Manolio TA: Use of sonography to evaluate carotid atherosclerosis in the elderly, the Cardiovascular Health Study. Stroke 1991, 22:1155-1163.

12. Tomiyama H, Yamashina A, Arai T, Hirose K, Koji Y, Chikamori T, Hori S, Yamamoto Y, Doba N, Hinohara S: Influences of age and gender on results of noninvasive brachial-ankle pulse wave velocity measurement a survey of 12517 subjects. Atherosclerosis 2003, 166:303-309.

13. Stickle D, Turk J: A kinetic mass balance model for 1,5-anhydroglucitol: applications to monitoring of glycemic control. Am J Physiol 1997, 273:E821-E830.

14. Kim WJ, Park CY: 1,5-Anhydroglucitol in diabetes mellitus. Endocrine 2013, 43:33-40.

15. Yamanouchi T, Ogata N, Tagaya T, Kawasaki T, Sekino N, Funato H, Akaoka L, Miyashita H: Clinical usefulness of serum 1,5-anhydroglucitol in monitoring glycaemic control. Lancet 1996, 347:1514-1518.

16. Yamanouchi T, Akanuma Y: Serum 1,5-anhydroglucitol (1,5 AG): new clinical marker for glycemic control. Diabetes Res Clin Pract 1994, 24:S261-S268.

17. Yoshioka K, Azukari K, Yoshida T, Kondo M: Rapid improvement of serum 1,5-anhydroglucitol concentrations after administration of a-glucosidase inhibitor. Diabetes Care 1997, 20:462.

18. Matsumoto K, Yano M, Miyake S, Ueki Y, Yamaguchi Y, Akazawa S, Tominaga $Y$ : Effects of voglibose on glycemic excursions, insulin secretion, and insulin sensitivity in non-insulin-treated NIDDM patients. Diabetes Care 1998, 21:256-260

19. Ouchi M, Oba K, Yamashita H, Okazaki M, Tsunoda M, Ohara M, Sekimizu K, Watanabe K, Suzuki T, Nakano H: Effects of sex and age on serum 1,5anhydroglucitol in nondiabetic subjects. Exp Clin Endocrinol Diabetes 2012, 120:288-295.

20. Monnier L: Is postprandial glucose a neglected cardiovascular risk factor in type 2 diabetes? Eur J Clin Invest 2000, 30(Suppl 2):3-11.

21. Kashiwagi A, Kasuga M, Araki E, Oka Y, Hanafusa T, Ito H, Tominaga M, Oikawa S, Noda M, Kawamura T, Sanke T, Namba M, Hashiramoto M, Sasahara T, Nishio
Y, Kuwa K, Ueki K, Takei I, Umemoto M, Murakami M, Yamakado M, Yatomi Y, Ohashi $\mathrm{H}$ : International clinical harmonization of glycated hemoglobin in Japan: From Japan Diabetes Society to National Glycohemoglobin Standardization Program values. Diabetol Int 2012, 3:8-10.

22. Nakatou T, Nakata K, Nakamura A, Itoshima T: Carotid haemodynamic parameters as risk factors for cerebral infarction in Type 2 diabetic patients. Diabet Med 2004, 21:223-229.

23. Nishiyama $Y$, Katsumata $T$, Otori $T$, Katayama $Y$ : Carotid hemodynamic parameters are useful for discriminating between atherothrombotic infarction and lacunar infarction. J Stroke Cerebrovasc Dis 2010, 19:441-449

24. Lee MY, Wu CM, Chu CS, Lee KT, Sheu SH, Lai WT: Association of carotid hemodynamics with risk of coronary heart disease in a Taiwanese population with essential hypertension. Am J Hypertens 2008, 21:696-700.

25. Koivistoinen $T$, Virtanen $M$, Hutri-Kähönen $N$, Lehtimäki $T$, Jula $A$, Juonala $M$, Moilanen L, Aatola H, Hyttinen J, Viikari JS, Raitakari OT, Kähönen M: Arterial pulse wave velocity in relation to carotid intima-media thickness, brachial flow-mediated dilation and carotid artery distensibility: the Cardiovascular Risk in Young Finns Study and the Health 2000 Survey. Atherosclerosis 2012, 220:387-393.

26. Yamashina A, Tomiyama H, Arai T, Hirose K, Koji Y, Hirayama Y, Yamamoto $Y$, Hori S: Brachial-ankle pulse wave velocity as a marker of atherosclerotic vascular damage and cardiovascular risk. Hypertens Res 2003, 26:615-622.

27. Mitchell GF, Hwang SJ, Vasan RS, Larson MG, Pencina MJ, Hamburg NM, Vita JA, Levy D, Benjamin EJ: Arterial stiffness and cardiovascular events: the Framingham Heart Study. Circulation 2010, 121:505-511.

28. Simons PCG, Algra A, Bots ML, Grobbee D, van der Graaf Y: Common carotid intima-media thickness and arterial stiffness: indicators of cardiovascular risk in high-risk patients: the SMART Study (Second Manifestations of ARTerial disease). Circulation 1999, 100:951-957.

29. Li CH, Wu JS, Yang YC, Shin CC, Lu FH, Chang CJ: Increase arterial stiffness in subjects with impaired glucose tolerance and newly diagnosed diabetes but not isolated impaired fasting glucose. J Clin Endocrionl Metab 2012, 97:E658-E662.

30. Huang $C L$, Chen MF, Jeng JS, Lin LY, Wang WL, Feng MH, Liau CS, Hwang BS, Lee YT, Su TC: Postchallenge hyperglycaemic spike associate with arterial stiffness. Int J Clin Pract 2007, 61:397-402.

31. Ledoux J, Werner ME, Brayden JE, Nelson MT: Calcium-activated potassium channels and the regulation of vascular tone. Physiology (Bethesda) 2006, 21:69-78.

32. Graier WF, Simecek S, Kukovetz WR, Kostner GM: High d-glucose-induced changes in endothelial Ca2+/EDRF signaling are due to generation of superoxide anions. Diabetes 1996, 45:1386-1395.

33. Cosentino F, Hishikawa K, Katusic ZS, Luscher TF: High glucose increases nitric oxide synthase expression and superoxide anion generation in human aortic endothelial cells. Circulation 1997, 96:25-28.

34. Ceriello A, Esposito K, Piconi L, Ihnat MA, Thorpe JE, Testa R, Boemi M, Giugliano D: Oscillating glucose is more deleterious on endothelial function and oxidative stress than mean glucose in normal and type 2 diabetic patients. Diabetes 2008, 57:1349-1354.

35. Azuma K, Kawamori R, Toyofuku Y, Kitahara Y, Sato F, Shimizu T, Miura K, Mine T, Tanaka Y, Mitsumata M, Watada H: Repetitive fluctuations in blood glucose enhance monocyte adhesion to the endothelium of rat thoracic aorta. Arterioscler Thromb Vasc Biol 2006, 26:2275-2280.

36. Ge QM, Dong Y, Zhang HM, Su Q: Effects of intermittent high glucose on oxidative stress in endothelial cells. Acta Diabetol 2010, 47(Suppl 1):97-103.

37. Kawano H, Motoyama T, Hirashima O, Hirai N, Miyao Y, Sakamoto T, Kugiyama K, Ogawa H, Yasue H: Hyperglycemia rapidly suppresses flowmediated endothelial-dependent vasodilation of brachial artery. J Am Coll Cardiol 1999, 34:146-154

38. Konukoglu D, Hatemi H, Ozer EM, Gonen S, Akcay T: The erythrocyte glutathione levels during oral glucose tolerance test. J Endocrinol Invest 1997, 20:471-475.

39. Xiang GD, Sun $H L$, Hou J, Yue L, Xu L: Acute hyperglycemia rapidly suppresses endothelium-dependent arterial dilation in first-degree relatives of type 2 diabetic patients. Exp Clin Endocrinol Diabetes 2008, 116:112-117.

40. Ceriello A, Bortolotti N, Crescentini A, Motz E, Lizzio S, Russo A, Ezsol Z, Tonutti L, Taboga C: Antioxidant defences are reduced during the ora glucose tolerance test in normal and non-insulin-dependent diabetic subjects. Eur J Clin Invest 1998, 28:329-333. 
41. Ceriello A, Bortolotti N, Motz E, Crescentini A, Lizzio S, Russo A, Tonutti L, Taboga C: Meal-generated oxidative stress in type 2 diabetic patients. Diabetes Care 1998, 21:1529-1533.

42. Yamanouchi T, Tachibana Y, Akanuma H, Minoda S, Shinohara T,

Moromizato H, Miyashita H, Akaoka I: Origin and disposal of 1,5-

anhydroglucitol, a major polyol in the human body. Am J Physiol 1992, 263:E268-E273.

doi:10.1186/1471-2261-13-11

Cite this article as: Watanabe et al:: Relationship between postprandial glucose level and carotid artery stiffness in patients without diabetes or cardiovascular disease. BMC Cardiovascular Disorders 2013 13:11.

\section{Submit your next manuscript to BioMed Central and take full advantage of:}

- Convenient online submission

- Thorough peer review

- No space constraints or color figure charges

- Immediate publication on acceptance

- Inclusion in PubMed, CAS, Scopus and Google Scholar

- Research which is freely available for redistribution 